\title{
ALGUNAS REFLEXIONES SOBRE LAS CONCEPCIONES ALTERNATIVAS Y EL CAMBIO CONCEPTUAL
}

\author{
OLIVA MARTÍNEZ, JOSÉ M. \\ Centro de Profesorado de Cádiz. Gravina s/n. 11003 Cádiz.
}

\begin{abstract}
SUMMARY
In this paper some recent contributions to the research of students conceptions in science are reviewed. The study analyses four principles that sustain a large part of the contributions emerged during the last years in this field: a) implicit structure, $b$ ) coexistence of ideas, $c$ ) limited homogeneity, and $d$ ) probability. Likewise, an analysis of possible processes that operate during the conceptual change is performed, by discussing on the effectiveness of the substitution of ideas and the conceptual conflict in order to achieve the conceptual change. On the base of these contributions, some implications for teaching and research in science education are pointed out.
\end{abstract}

\section{INTRODUCCIÓN}

Si analizamos las fuentes de desarrollo de la visión constructivista de la enseñanza-aprendizaje de las ciencias y, dentro de ella, del enfoque de las concepciones alternativas, podemos distinguir valiosas aportaciones. Particularmente, cabe destacar las contribuciones provenientes del campo de la psicología evolutiva y de la instrucción, y todo el cuerpo de conocimientos desarrollados en torno a la epistemología y la historia de las ciencias. Todas estas aportaciones han fermentado de un modo conjunto y se han integrado en el campo de la didáctica de las ciencias, constituyéndose en un auténtico paradigma de investigación.

Desde finales de los ochenta se aprecia además toda una corriente interna de profundización en el estudio de las concepciones fruto de la incorporación de nuevas aportaciones derivadas, entre otras, de cuatro líneas de estudios: a) aquéllos dirigidos a investigar las propiedades de las concepciones (coherencia, consistencia, persistencia, etc.); b) los trabajos sobre modelización del pensamiento causal; $c$ ) aquellos otros que han introduci- do fundamentos teóricos procedentes de la psicología cognitiva y de la inteligencia artificial; y $d$ ) los estudios recientes que están replanteando las bases epistemológicas de los modelos de cambio conceptual. En este trabajo pasamos revista a algunas de las aportaciones más importantes surgidas como consecuencia de estos nuevos influjos, así como a fundamentar los puntos de vista defendidos desde cada una de las posturas.

\section{SOBRE LA NATURALEZA DE LAS CON- CEPCIONES}

En relación con la naturaleza de las concepciones, de las aportaciones mencionadas se desprenden varios aspectos que creemos de interés señalar. Éstos podrían formularse sobre la base de cuatro principios básicos, como son el de estructuración implícita, el de diversidadcoexistencia, el de sistematicidad - homogeneidad limitada y el de probabilidad. 
Las concepciones como formas de razonamiento implícitas

Frente a la condición de observable que se le ha solido atribuir, cada vez cobra mayor fuerza la idea de otorgar al conocimiento intuitivo una naturaleza interna y potencial, en la cual las concepciones que se detectan no son sino la punta del iceberg que asoma por encima de la superficie del agua. Según este punto de vista, las ideas que los alumnos manifiestan y que se delimitan directamente a través de los cuestionarios y entrevistas, no serían en sí mismas las concepciones que éstos poseen sino sus manifestaciones externas mediatizadas por factores tales como el contexto o la tarea. De ahí que pueda existir un cierto grado de indeterminación entre lo que observamos en las respuestas y el conocimiento que realmente se encuentra detrás (Dykstra et al., 1992). Ni todo lo que se cree descubrir en las respuestas de los alumnos responde a ideas previas en el sentido literal del término, ni todo el conocimiento interno se llega a exteriorizar de un modo directo e inequívoco a través de las concepciones que como investigadores llegamos a extraer.

Por ejemplo, durante los últimos años ha empezado a emerger en psicología un nuevo enfoque en el análisis del pensamiento intuitivo de sentido común, como punto de encuentro entre la psicología cognitiva, y otras dos tradiciones de pensamiento e investigación, como son la psicología de la cognición social y el estudio de las representaciones colectivas. Nos referimos al ámbito de reflexión y análisis que se ha desarrollado en torno al concepto de teoría implícita como término que describe todo el trasfondo que se sitúa tras las creencias que mantienen las personas en su pensamiento ordinario.

Las características de las teorías implícitas, como forma de conceptualización de las representaciones de los individuos y de sus teorías personales, han sido resumidas por Rodríguez y González (1995) y guardan un importante paralelismo con las características postuladas para las concepciones alternativas en el ámbito de los contenidos científicos. No es casual, por tanto, que autores como Pozo (Pozo et al., 1992, 1995) aludan al movimiento de las teorías implícitas, como núcleo de fundamentación teórica para el estudio de los conocimientos previos y de las concepciones precientíficas de los estudiantes, y que este enfoque venga constituyéndose últimamente como referente en algunas investigaciones experimentales orientadas a comprender mejor las características de las concepciones de los alumnos.

Las diferencias entre las teorías científicas y las teorías implícitas han sido analizadas por diversos autores (Rodríguez et al., 1993). En cierta forma algunas de ellas son análogas a las que se describen a la hora de hablar de diferencias entre expertos y novatos en un área de conocimiento, y de ahí que se contemple el aprendizaje de contenidos científicos como un proceso de explicitación de las teorías personales y su transformación hacia posiciones más próximas a la ciencia formal. Frente al punto de vista adoptado por la mayoría de los autores, de atribuir a las ideas el estatus de creencias basadas en observaciones empíricas puntuales aplicables sólo a casos particulares, desde aquí se asume que el pensamiento intuitivo acerca de los fenómenos científicos es de naturaleza causal. Se retoma además la distinción entre competencia y actuación de los sujetos en la resolución de tareas, que resulta característica como sabemos de los modelos computacionales y de las teorías neopiagetianas, y utiliza la noción de activación como un elemento central del proceso de deliberación y toma de decisiones por parte del alumno. Se podría disponer de un determinado esquema y no activarlo para una situación concreta aunque sí en otra, y de ahí que el conocimiento se considere un componente latente que no siempre es exteriorizable. Se abre la posibilidad, por tanto, de que un mismo alumno active concepciones diferentes en distintas ocasiones, lo cual no puede ser más que por la existencia de una cierta competitividad entre esquemas distintos que pueden convivir en el pensamiento.

\section{Posibilidad de coexistencia de concepciones diferentes sobre un mismo tópico}

Los estudios sistemáticos llevados a cabo sobre la influencia del contexto en el uso de concepciones a través de distintas tareas constituyen un buen ejemplo que ilustra uno de los tipos de datos empíricos que han servido de apoyo a la hora de defender la hipótesis constructivista de las concepciones espontáneas. En efecto, algunas autoras y autores (Clough y Driver, 1986; Jiménez-Aleixandre y Fernández, 1989; Oliva, 1994) han utilizado los criterios de consistencia como argumento para defender la existencia de concepciones firmemente asumidas por los alumnos, frente a la hipótesis trivial que ha visto en ellas simples contestaciones ad hoc generadas para salir al paso de las cuestiones formuladas. A pesar de conseguir sus objetivos, parcialmente, los trabajos de este tipo han mostrado una realidad más compleja de la que en principio se esperaba, con niveles estadísticamente significativos de consistencia en los esquemas usados, pero al mismo tiempo, también, con variaciones importantes en las respuestas emitidas. Si bien las ideas de los alumnos no responden a respuestas aleatorias, su existencia tampoco se explica satisfactoriamente desde una visión constructivista centrada en la hegemonía de esquemas de conocimiento únicos perfectamente establecidos y asentados. Ello ha contribuido a abrir un debate en torno al diferente grado de compromiso cognitivo que el alumno mantiene con sus concepciones en distintos casos. En unas ocasiones podrían ser el reflejo de un conocimiento interno verdaderamente estable, mientras que en otros podrían estar condicionadas por una forma excesivamente directiva de plantear las tareas que sirven para su detección.

Autores como Marton (1981) ya mencionaban hace unos años la existencia de variaciones en las concepciones para un mismo fenómeno, no sólo entre niños distintos sino incluso a nivel individual en función del contexto involucrado. Según esta hipótesis, que ha sido contrastada con posterioridad en los trabajos sobre coherencia y consistencia en las ideas, las concepciones no serían 
consecuencia de los rasgos de un individuo, sino que son características de las relaciones entre el individuo, el contenido y el contexto. Linder (1993) denomina a este fenómeno dispersión conceptual, y muchos autores se refieren a él cuando sugieren la posibilidad de que puedan coexistir en un mismo alumno distintas concepciones que compiten ante una situación determinada (Maloney y Siegler, 1993).

Al hablar de coexistencia de concepciones no sólo nos referimos a que, después de la instrucción, los alumnos compartan una visión científica y una visión intuitiva previa a la enseñanza. Nos referimos incluso a que, ya desde una fase anterior al aprendizaje escolar, un mismo alumno pudiera compartir diversos esquemas alternativos sobre un mismo tópico que rivalizan y compiten entre sí. Las reglas que rigen la influencia del contexto en la activación y competencia de esos esquemas han sido ya apuntadas por diversos autores (Pozo et al., 1991; Maloney y Siegler, 1993), pero por problemas de espacio no vamos a mencionarlas aquí, aunque sí analizaremos algunos aspectos relativos a la homogeneidad y coherencia de las ideas expresadas en distintas situaciones.

\section{Existencia de un cierto nivel de sistematicidad u homogeneidad en las concepciones a través de distintos ámbitos}

En los últimos años se ha realizado un esfuerzo considerable por analizar el grado de organización interna de las concepciones de los alumnos. Estos estudios han evidenciado que, si bien las concepciones no tienen un nivel de homogeneidad y estructuración semejante al que poseen las teorías científicas o como el que le atribuía el enfoque piagetiano de las operaciones formales, tampoco son tan fragmentarias y dispersas como suponían los estudios descriptivos realizados en los últimos quince años (Pozo et al., 1992). Se ha evidenciado, por ejemplo, que la capacidad de los alumnos para generalizar es bastante limitada, pero aun así se aprecia un cierto grado de homogeneidad en las ideas que surgen a través de distintos contextos y tareas (Clough y Driver, 1986; Jiménez-Aleixandre y Fernández, 1989; Oliva, 1996).

El enfoque teórico propuesto por Pozo y otros en sus trabajos (1992) defiende una visión intermedia entre una y otra y establece la existencia de una serie de estructuras con un cierto grado de generalidad que explicaría las parciales regularidades encontradas en el pensamiento intuitivo en diferentes ocasiones. Se comportarían como sesgos o formas de razonamiento transversales (Pintó et al., 1996) en una dirección próxima a las propuestas realizadas por Viennot en sus trabajos. Desde aquí se explica la comunalidad observada en las concepciones en distintos dominios científicos aludiendo a estructuras latentes de este tipo que se repiten parcialmente de una ocasión a otra.

Sin ánimo de ser exhaustivos, a continuación retomamos algunos ejemplos de tales esquemas genéricos aprovechando las aportaciones de diversos autores (Andersson,
1986; Rozier y Viennot, 1991; Viennot y Kaminski, 1991; Pozo et al., 1991; Stavy y Tirosh, 1996; Tirosh y Stavy, 1996; Watts y Taber, 1996):

a) Reducción en el número de variables. Una causa sólo tiene un efecto.

b) Ausencia de reciprocidad o retroacción en las relaciones.

c) Tendencia a explicar los cambios y no los estados. En este último caso, se suelen emplear argumentos del tipo es natural, es normal o es de sentido común.

d) Escaso nivel de cuantificación de las relaciones. Tendencia a aportar, a lo sumo, argumentos de tipo semicuantitativo del tipo a más A... más $B$, o del tipo cualquier cosa puede ser siempre dividida en dos.

e) Ausencia de conservación en las transformaciones.

f) Atención a las propiedades más llamativas y superficiales de los sistemas más que a las características funcionales a la hora de darles una explicación.

En su conjunto esta perspectiva está en sintonía con otro enfoque teórico que utiliza los modelos mentales de la psicología computacional como instrumento de representación del conocimiento. En realidad no existe un único enfoque basado en el uso de modelos mentales como forma de representación y teorización en elámbito computacional. En la propia enseñanza de las ciencias aparecen diferentes propuestas en este sentido con objeto de interpretar la naturaleza de las concepciones de sentido común acerca de la ciencia, como lo muestra la revisión reciente realizada por Moreira (1997). Una de las aportaciones pioneras en este terreno la encontramos en los trabajos de Gutiérrez basados en el modelo mecánico de Kleer y Brown (Gutiérrez y Ogborn, 1992; Gutiérrez, 1990). Coincidiendo con otros enfoques considera que los mecanismos que el sujeto utiliza para explicar y predecir el comportamiento de los sistemas físicos son de carácter causal, es decir, buscando causas y efectos, y que el aprendizaje se verifica mediante construcción de nuevos modelos mentales y mediante la reconstrucción de los ya existentes para hacerlos más útiles. Dicha utilidad vendría regida por una serie de características, como son su consistencia, en cuanto a propiedad de carecer de contradicciones internas; su correspondencia, como cualidad de acercar sus predicciones al comportamiento del mundo real; y su robustez, como capacidad del modelo para adaptarse a situaciones novedosas para las que no estaba previsto en principio.

Los enfoques basados en el uso de modelos mentales vienen cobrando cada vez mayor fuerza y prueba de ello lo tenemos en que ya algunos trabajos empiezan a optar por ellos a la hora de analizar los datos obtenidos acerca de las concepciones de los alumnos (Vosniadou, 1994; Harrison y Treagust, 1996; Blanco y Prieto, 1997; Núñez y Banet, 1996; Greca y Moreira, 1997). Por lo general sugieren una visión en la que las concepciones no aparecen como componentes fragmentarios que funcionan y 
trabajan de una forma desordenada, sino que lo hacen de una manera estructurada y con un cierto grado de lógica y coherencia. Tratan de aportar pautas que ayuden a comprender cómo los alumnos coordinan distintas piezas de conocimiento en un mismo dominio para dar un significado global a los fenómenos que trata de interpretar de un modo causal.

Pero los modelos mentales intuitivos no son tan precisos, consistentes y completos como lo son los modelos científicos, son simplemente funcionales (Moreira, 1997). Por ello, la coherencia que desde aquí se postula es sólo parcial y de ahí la necesidad de asumir una perspectiva probabilística del razonamiento como trataremos a continuación de fundamentar.

\section{Las concepciones como constructos de tipo proba- bilístico}

La mayoría de los estudios sobre concepciones alternativas han optado por un enfoque determinista en el que las ideas se consideran el resultado directo e inequívoco de la ecología conceptual. Se supone que, si fuéramos capaces de conocer en toda su extensión cuál es la estructura cognitiva de un sujeto, podríamos predecir cuál sería la concepción que mantendría en un contexto y situación determinada. Según esta hipótesis, las concepciones que los sujetos exteriorizan en sus respuestas y explicaciones están predeterminadas desde una fase anterior a la formulación de las tareas a través de las cuales afloran, y las distribuciones de frecuencias que se obtienen en las respuestas se interpretan como el conjunto de proporciones de alumnos que pueden encasillarse en cada una de las opciones.

Frente a esta posición, empieza a emerger un enfoque diferente según el cual la asunción o no de un determinado esquema y su posibilidad de activación no constituyen una cuestión de «todo o nada» sino de grado, y que el proceso de reflexión y toma de decisiones se rige por criterios de la lógica probabilística y conlleva siempre un cierto nivel de incertidumbre en su resultado. Así, por ejemplo, Monk (1995) en un artículo reciente proponía diversos principios de la ciencia que pueden ser útiles como herramienta de trabajo en el estudio de las concepciones de los alumnos. Entre ellos incluía la probabilidad estadística como noción que permite conectar distintos niveles de análisis para un fenómeno, como son, por ejemplo, los niveles macroscópico y microscópico que operan en física en el estudio de un gas, o también los niveles más elementales y los de más alto nivel que operan en la estructura conceptual de un alumno:

«Cuando recopilamos las creencias de los alumnos acerca de un fenómeno físico particular, los investigadores constructivistas llevamos a cabo una tarea similar desde el punto de vista analógico a la de investigar la biografía de una partícula individual de un gas [...] De la misma manera que no es posible decir qué es lo que hace una partícula de un gas en un momento dado, tampoco es posible decir qué es lo que un alumno está pensando.» (Monk, 1995, p. 568).
El principal tipo de datos empíricos a favor de este enfoque lo encontramos de nuevo en los estudios sobre dependencia del contexto señalados antes y también en otros estudios recientes sobre estabilidad de las concepciones. Así, por ejemplo, en un trabajo realizado por nosotros (Oliva, 1998a) se ha podido comprobar cómo alrededor del $40 \%$ de alumnos de $1^{\circ}$ de BUP utiliza concepciones intuitivas diferentes acerca de fenómenos relacionados con el ámbito de la mecánica, al repetir la prueba en un diseño test-retest sin haber transcurrido instrucción intermedia en temas de física. Como se recordará, en el anterior sistema educativo, los alumnos de este nivel de enseñanza no estudiaban física y química, por lo que puede afirmarse que la variación detectada en las respuestas es ajena al efecto de instrucción. Por otra parte, además, las diferencias mencionadas se observan sólo si los resultados se analizan desde un punto de vista individual pero no cuando éstos se comparan con una perspectiva muestral. Ello indica que la variabilidad se debe a un efecto de tipo estadístico y no a un fenómeno de olvido o de cambio permanente. Este resultado parece apoyar la visión probabilística de la que hablamos ilustrando el grado de variabilidad que las concepciones pueden llegar a tener incluso dentro de un mismo individuo.

El propio enfoque de las teorías implícitas del que hablamos antes reconoce el carácter probabilístico de las concepciones alternativas, otorgando al pensamiento intuitivo una naturaleza difusa similar a la que asignan a los conceptos las teorías computacionales, en contraposición con el conocimiento científico que resulta claramente definido y unívoco. En consecuencia, la respuesta de un alumno a una cuestión concreta no estaría sujeta a una lógica determinista, fijada previamente a la formulación de la misma, sino que sería el resultado de un proceso de decisión entre diversos argumentos y opciones que mantienen un determinado estatus (Hewson y Beeth, 1995). Las frecuencias de respuesta a una pregunta dada no serían un reflejo de la proporción de alumnos que mantienen una u otra idea, sino de la probabilidad de que el alumno medio active y utilice una cierta concepción en un momento y contexto dados.

Esta indeterminación e incertidumbre afecta al parecer a todos los procesos superiores, desde la memorización hasta el razonamiento y el aprendizaje, y encuentra fundamento en diversas posiciones teóricas. En primer lugar, hemos de recordar el carácter difuso que otorgan las teorías computacionales a las categorías conceptuales y los resultados del aprendizaje. Multitud de aspectos relativos a ellas escapan a la dicotomía de todo o nada, siendo más bien una cuestión de grado. Ejemplo de ello lo es la pertenencia o no de cada noción a otra más inclusora dentro de la jerarquía conceptual, la certeza de las reglas de inferencia que permiten relacionar a unos conceptos con otros, o los criterios de categorización y clasificación de los contextos fenomenológicos. Así mismo, hemos de mencionar los estudios teóricos y de implementación computacional en el ámbito de los sistemas expertos, en los que el análisis de la propagación de la incertidumbre en el razonamiento constituye un problema habitual. 
Epistemológicamente podríamos contextualizar esta idea dentro de las mismas corrientes que dieron lugar al indeterminismo en la física de principios de siglo y que llevaron, por ejemplo, a sustituir la noción de certeza absoluta por la de probabilidad en los modelos cuánticos. También podríamos asociar estas ideas con aquellas provenientes de la teoría del caos, la cual parte del rechazo a la visión mecanicista y positivista clásica que asegura la posibilidad de conocer el estado final de la evolución de un sistema una vez conocidas con precisión cuáles son sus condiciones iniciales. Cuando varias reglas de inferencia actúan simultáneamente reforzando o contraponiendo sus efectos y actúan múltiples variables de fondo que interaccionan entre sí, a veces de una forma desconocida, el panorama puede considerarse lo suficientemente complejo para que el resultado pueda catalogarse dentro de ciertos límites como de impredecible. En esta línea, algunas visiones recientes (Luffiego et al., 1994) equiparan al sistema cognitivo con otros sistemas dinámicos complejos y basan el paralelismo en la naturaleza no lineal de los procesos que en ellos tienen lugar.

En resumidas cuentas puede decirse que el principio de probabilidad, junto con los otros tres que hemos fundamentado antes, nos ayudan a interpretar el comportamiento de los alumnos en la formulación de concepciones e ideas espontáneas en el ámbito científico. Como es de esperar estos principios tienen importantes repercusiones en la manera de entender el aprendizaje a través del cambio conceptual, aspecto al que vamos a referirnos a continuación.

\section{SOBRE LOS MECANISMOS DE CAMBIO CONCEPTUAL}

Las aportaciones más relevantes y actuales en este terreno se han centrado en el debate en torno a los siguientes aspectos: la existencia de más de un mecanismo de cambio conceptual, el carácter gradual y paulatino de dicho proceso, la naturaleza más o menos general o específica del proceso de cambio, el cuestionamiento de la sustitución de ideas como mecanismo de aprendizaje y las críticas vertidas sobre el conflicto conceptual como estrategia idónea para el cambio en las ideas.

\section{Existencia de más de un mecanismo de cambio con- ceptual}

Son diversas las causas que justifican hoy una visión del cambio conceptual como proceso complejo y diverso en el que cabe más de un mecanismo. Ya en los primeros modelos de cambio aparece una visión dual de dicho proceso. Así, frente a la visión radical que propugna el modelo original de Posner y otros (1982), centrado principalmente en los fenómenos de acomodación en el aprendizaje, Hewson (1981) -uno de los coautores del modelo-propone una modalidad diferente que amplía la anterior para el caso en el que las nuevas ideas no sean totalmente incompatibles con la visión mantenida previamente por el alumno. A esta modalidad, la denomina captura conceptual en la cual los procesos de asimilación juegan un papel más importante.

Por otra parte, en algunas teorías computacionales como la teoría de los esquemas de Norman y Rumelhart, se nos habla también acerca de diferentes mecanismos de elaboración del conocimiento señalándose la existencia de períodos de crecimiento y ajuste de esquemas y períodos de reestructuración en el aprendizaje. Precisamente es a partir de la teoría de los esquemas sobre la cual Luffiego y otros (1994) fundamentan su modelo de evolución conceptual. Según estos autores, los sistemas conceptuales evolucionan en cadena, de forma que en cada etapa se incorpora nueva información cuyo producto puede resultar diferente de la mera suma al estado anterior de la información añadida. En las fases que llaman de estabilidad el sistema adopta un comportamiento no lineal poco pronunciado, mostrándose resistente a los cambios a gran escala y verificándose en ellos solamente procesos de acumulación y refinamiento sobre un captador conceptual que sirve de ancla para la información que entra. Pero llegado el caso, el sistema puede volverse inestable, con lo que el carácter no lineal del proceso de adquisición de información se agudiza y el sistema evoluciona a partir de un captador caótico que lleva al fenómeno a convertirse en impredecible. En esta fase, cantidades mínimas de información o pequeñas fluctuaciones en las condiciones iniciales pueden verse amplificadas en un fenómeno parecido a los fenómenos físicos de resonancia. Y estos cambios podrían adquirir significatividad dentro del esquema conceptual, cristalizando en un nuevo sistema de ideas.

Esta multiplicidad en la modalidad de cambio parece también acorde con los distintos mecanismos de aprendizaje postulados por otros autores. Así, de una parte, Carey (1991) distingue entre procesos de reestructuración fuerte y de reestructuración débil, basando su distinción en la posibilidad de verificarse un cambio que afecte al compromiso ontológico del individuo o simplemente a la adquisición de nuevos conocimientos y a la diferenciación e integración de nociones que ya posee. Para Chi (1992, citado por Dagher, 1994), ese cambio ontológico se podría sustentar sobre la distinción de tres categorías de conocimiento como son la materia, los sucesos y las abstracciones. Según él, cuando el cambio implica una reorganización dentro de cada categoría tenemos un cambio conceptual normal, mientras que, cuando el cambio sucede a través de distintas categorías, tenemos un cambio conceptual radical.

También esta visión diversa del cambio en las ideas es acorde con el punto de vista adoptado por Vosniadou (1994). Para esta autora, el cambio conceptual se interpreta como una modificación progresiva en los modelos mentales que posee el sujeto, modificación que puede ser generada por un proceso de enriquecimiento de información a partir de la estructura conceptual existente o bien por una revisión o cambio de las creencias y presupuestos de partida. 
A pesar de la variabilidad observada en la terminología usada por los diversos autores, las dos modalidades de cambio sugeridas en los diferentes casos parece ajustarse bastante bien a la de acomodación y asimilación apuntadas por Piaget. Mientras que, en la captura, crecimiento de esquemas, enriquecimiento, ajuste, reestructuración débil o cambio normal, predominarían los procesos de asimilación y cambio parcial en el conocimiento, en las modalidades de revisión, reestructuración fuerte -o simplemente reestructuración-o cambio radical, predominarían los de acomodación. En lo que no parecen ponerse de acuerdo los distintos autores es en el alcance que otorgan al significado de la noción de cambio conceptual. Así, mientras algunos de ellos (Hewson, 1981; Hewson y Hewson, 1992; Chi, 1992; Dagher, 1994) extienden esta condición para ambos mecanismos, autoras como Carey (1991) sólo atribuyen a la primera la categoría de un auténtico cambio conceptual.

Pero el panorama se revela hoy aún más complejo si cabe, y en los trabajos más recientes Hewson distingue hasta tres variedades de cambio conceptual (Hewson y Hewson, 1992). Junto a la noción de cambio como «sustitución de ideas» y a la noción de captura conceptual, contempladas ambas en trabajos anteriores, este autor reconoce una tercera modalidad a la que denomina canje o intercambio (exchange), en la que la nueva concepción es preferida a la anterior aunque no logra extinguirla y termina conviviendo con ella. En esta modalidad, la sustitución no es permanente sino ocasional, quedando ambas estructuras en estado latente. No se alcanza un cambio por mutación de unas ideas en otras, ni tampoco las nuevas ideas se integran dentro de las anteriores. Más bien sucede que las nuevas ideas se diferencian de las otras manteniendo con ellas un comportamiento coexistente y de uso alternante.

Todas estas consideraciones se refieren a los mecanismos del cambio o posibles cambios en las ideas y no tanto a la escala de tiempo en la que se verifican las transformaciones. Cuando dicha escala temporal es tenida en cuenta los diversos tipos de procesos, no aparecen como realidades tan fácilmente diferenciables.

\section{El cambio conceptual como cambio profundo pero que opera de un modo gradual}

En el propio modelo original de Posner y otros, sus autores reconocen que el proceso de acomodación que exige el cambio conceptual tiene un carácter gradual que se efectúa poco a poco, siendo escasamente probable que los estudiantes capten desde el primer momento cualquier teoría en su totalidad y sus implicaciones respecto al mundo. De hecho, Thagard (citado por Dagher, 1994) ha analizado detenidamente cuáles son los mecanismos a través de los cuales se verifica el cambio conceptual, llegando a distinguir hasta nueve grados distintos. Por ello, el autor concluye la existencia de una gradación continua en la complejidad del cambio conceptual, lo que para él supone la imposibilidad de hablar del cambio conceptual como una cuestión de todo o nada.
Desde el punto de vista de las teorías computacionales del conocimiento, podríamos decir que la intensidad de cada vínculo en la compleja red de relaciones que integran la estructura de conocimientos, no varía de forma súbita sino que lo hace de un modo continuo a través de toda una gama de probabilidades que no suele adoptar como valores extremos. Se debería hablar, pues, a la hora de concebir el proceso de reestructuración, de un mecanismo gradual que opera a través del debilitamiento progresivo de ciertas relaciones entre conceptos y de ciertas reglas de inferencia de naturaleza intuitiva, y del reforzamiento paulatino de relaciones y reglas más acordes con el punto de vista científico.

Epistemológicamente, algunos autores sostienen también una visión evolutiva del desarrollo del conocimiento científico. Entre ellos cabría destacar al propio Toulmin, quien rechaza la división entre ciencia normal y revolucionaria y adopta una visión evolutiva de los procesos de cambio científico, o a Bachelard y toda la tradición de la escuela francesa, que centran su atención, no tanto en la estructura en su conjunto de las teorías científicas como en la óptica que ofrece el estudio del significado de los conceptos. Según estos puntos de vista, se contempla que parte de los rasgos y atributos importantes que caracterizan los conceptos de una teoría, puedan heredarse a partir del significado que tienen en otras formulaciones predecesoras; y que, llegado el caso, podría incluso producirse una cierta trasferencia de significados entre teorías consideradas antagónicas. De esta forma, la adquisición de conceptos podría verse también como un proceso de incorporación y eliminación paulatina de ciertos atributos partiendo de una noción inicial que, en el fondo, no se vería totalmente modificada.

Incluso la perspectiva del cambio como algo gradual parece estar en consonancia con posturas epistemológicas más recientes como las de Laudan (Duschl y Gitomer, 1995). Mientras autores como Khun y Lakatos asumen un enfoque radical del cambio en las ideas y una visión jerarquizada del conocimiento en la que lo conceptual prima sobre los procedimientos e intenciones, para Laudan, las teorías, métodos y objetivos de las ciencias forman un entramado complejo y articulado en el que, más que una relación de subordinación entre esos elementos, se verifica una relación de interdependencia e influencia mutua. De ahí que una transformación en el conocimiento requiera de un cambio sustantivo a través de distintas instancias, lo que difícilmente va a poder verse si no es por medio de una cierta reciprocidad y continuidad en el proceso. Esta otra visión epistemológica tiene importantes repercusiones en la enseñanza de las ciencias, y Duschl y Gitomer (1995) la utilizan para sugerir una perspectiva evolutiva gradual del cambio conceptual:

«Un modelo evolutivo del crecimiento científico ayudaría a estudiantes y enseñantes a organizar los marcos conceptuales de la ciencia y a alcanzar un estado en el que los estudiantes sean capaces de evaluar el grado legítimo de duda asociado a declaraciones de conocimiento científico.» (Duschl y Gitomer, 1995; p. 122). 
Psicológicamente, incluso esta visión gradual del proceso de cambio encuentra un soporte teórico en las posturas defendidas por Vygotski. En efecto, a veces los alumnos podrían no llegar a hacer un uso efectivo de las concepciones científicas que se enseñan en la escuela, no porque las desconozcan o carezcan de ellas, sino porque se encuentran localizadas dentro de lo que ese autor llama zona del desarrollo potencial. En tales casos, las nociones implicadas no podrían ser usadas de un modo autónomo por los individuos, pero podrían ser susceptibles de un desarrollo condicionado por la recepción de cierta ayuda a través del profesor, los propios compañeros o el mismo material de aprendizaje. Esas ideas existirían, por tanto, ya en un principio, por lo que el proceso de aprendizaje cumpliría más en esos casos la función de un germen favorecedor e impulsor de las mismas que la del precursor de una transformación revolucionaria a gran escala.

Otra evidencia a favor de la idea de cambio gradual la encontramos en datos procedentes de investigaciones emprendidas en la propia didáctica de las ciencias. En efecto, las investigaciones llevadas a cabo sobre la evolución en las ideas de los estudiantes (Nussbaum, 1989) y aquellas otras emprendidas sobre la influencia del contexto parecen poner de manifiesto que las ideas intuitivas y esquemas alternativos evolucionan poco a poco, de una forma progresiva, más que de un modo brusco y revolucionario como lo hacen los cambios de paradigmas a lo largo de la historia de las ciencias. La mayoría de las veces las ideas cambian de un modo paulatino y gradual siendo frecuente la aparición de concepciones híbridas entre las de sentido común y las científicas (Galili y Bar, 1997). Licht (1987), por ejemplo, ha observado que, al tratar de aplicar estrategias de cambio conceptual en temas introductorios de electricidad, obtiene resultados sólo moderadamente optimistas, apreciando un excesivo desnivel entre el nivel intuitivo en el que se mueve el pensamiento inicial de los alumnos y el nivel teórico y explicativo de las nuevas ideas que les son presentadas. Como consecuencia de ello, y con objeto de soslayar la dificultad encontrada, el mencionado autor propone el establecimiento de una fase intermedia entre ambos en la cual los alumnos puedan descubrir, de forma orientada, reglas y regularidades a través de sus propias observaciones, y describir los fenómenos en términos cualitativos y cuantitativos sin esperar un cambio sustancial en la naturaleza de las ideas mantenidas. En esa fase, los alumnos podrían clasificar, comparar y relacionar objetos o conceptos empleando incluso un lenguaje no científico. Su objetivo fundamental sería el de lograr establecer en sus ideas intuitivas una red lógica de interconexión y diferenciación entre conceptos que les permitiera realizar algunas generalizaciones y argumentaciones acerca de fenómenos. Esta posición es muy similar a la expuesta por Linjse (1990), el cual considera que, al menos en ciertos tópicos, como el de energía, no se debería planificar la enseñanza con el fin de destruir las ideas de los alumnos, sino con la idea de propiciar en ellos un uso más flexible de las mismas. Desde este punto de vista, el cambio conceptual no siempre implicaría un cambio hacia la verdad científica, sino que podría también involucrar cambios parciales hacia posi- ciones intermedias que sirviesen de puente entre la visión intuitiva de partida y la noción científica que se desea enseñar.

En resumen, podemos decir que el cambio conceptual, más que como un proceso súbito y radical, queda mejor explicado como un debilitamiento del estatus de las concepciones previas y en un fortalecimiento simultáneo del estatus de las nuevas (Hewson y Thorley, 1989). Existe, pues, una cierta continuidad longitudinal en la evolución del pensamiento que, como veremos a continuación, algunos autores la extienden a una supuesta conexión lógica en el desarrollo de distintas concepciones.

\section{El cambio conceptual como algo que va más allá del cambio en conocimientos específicos}

Como es de suponer, una posición intermedia para el estatus de las concepciones entre el que le otorga el enfoque de las operaciones formales y el que le asigna el enfoque de las concepciones alternativas conlleva también implicaciones importantes en la manera de comprender el cambio conceptual.

De una parte, según el enfoque de las operaciones formales, dicho proceso lleva asociado una transformación en las estructuras generales de pensamiento, desde un estadio de pensamiento concreto basado en los aspectos más claramente perceptibles a otro de pensamiento formal apto ya para comprender los aspectos más abstractos de la ciencia. Cambiar las ideas de los alumnos sobre el concepto de fuerza, sobre circuitos eléctricos o sobre la naturaleza de los gases, por ejemplo, implicaría un cambio global en las estructuras mentales de razonamiento a través del cambio en las operaciones mentales en las que tienen su origen, aspecto al que debería ir dirigida la instrucción.

De otra, en el enfoque de las concepciones alternativas, el cambio se contempla como un proceso parcial que sucede en cada tópico concreto, sin que exista un nivel de generalidad en el conocimiento que justifique un tratamiento global del mismo con independencia de los contenidos implicados. Según esta visión, el cambio en el conocimiento sólo se puede verificar si la instrucción se dirige directamente a los contenidos más íntimamente relacionados con cada tema particular, dado que una modificación en una parte de la estructura cognitiva no tendría por qué conllevar un cambio simultáneo en otras partes distintas.

Desde la óptica por la que apuesta el enfoque de las teorías implícitas cabe suponer un comportamiento intermedio ente una y otra opción, pudiéndose llegar a esperar, dentro de ciertos límites, cambios en algunas concepciones una vez que se han cambiado otras con las que comparte algunos elementos de las estructuras implícitas. Así, pues, superar las concepciones erróneas desde el punto de vista de la ciencia escolar conllevaría algo más que un cambio local en contenidos específicos. Comportaría un cambio en las teorías implícitas en las 
que subyacen las respuestas y contestaciones de los alumnos y, en consecuencia, de un cambio en las estructuras mentales que con carácter más o menos general se encuentran tras ellas.

En favor de ello diremos que existen diversas evidencias que corroboran este punto de vista, empezando por los propios datos comentados antes sobre el nivel de generalidad en el uso de concepciones a través de distintos contextos. Además, existen diversos estudios que han mostrado que, aunque el aprendizaje está altamente influido por el contexto, las estrategias cognitivas involucradas en la generación y contraste de hipótesis y en la evaluación de resultados, pueden ser generalizadas de unos contextos a otros (Khun et al., 1992; Schauble et al., 1991; Keys, 1997), con lo cual el aprendizaje en una parte del sistema cognitivo podría influir en otras aparentemente distintas. De hecho, a pesar de las múltiples críticas que el enfoque de las operaciones formales ha recibido en los últimos años, no carece de un cierto soporte empírico. Nos referimos concretamente a los estudios que han evidenciado la existencia de una importante correlación entre el rendimiento en pruebas generales de pensamiento formal y el nivel de cambio conceptual alcanzado en temas concretos, como en genética y evolución (Lawson y Thompson 1988), en la interpretación cinético-molecular del calor (Rogan, 1988) y en el estudio de la energía (Trumper y Gorsky, 1993) o de la mecánica (Acevedo et al., 1989; Oliva, 1994).

También podemos destacar los trabajos de Shayer y Adey en los que describen el diseño y los resultados de su proyecto sobre aceleración del desarrollo cognitivo CASE (Cognitive Acceleration through Science Education) (Adey y Shayer, 1990; Shayer y Adey, 1992; Adey, 1992). Estos autores advierten de las dificultades que existen para trasladar las habilidades de razonamiento de unos esquemas lógicos a otros, e incluso de una tarea a otra dentro de un mismo esquema. Pero muestran también que es posible obtener algunos resultados al respecto cuando las estrategias formales no se instruyen directamente sino que lo hacen de un modo contextualizado a través de las clases de ciencias. En efecto, como consecuencia de esta serie de trabajos, se deducen, entre otras, dos conclusiones de interés: a) al cabo de dos años los alumnos del grupo experimental que participaban en el proyecto superaban ya en sus destrezas cognitivas a sus homólogos del grupo control; y $b$ ) esta mejora se traducía incluso a más largo plazo en un rendimiento en ciencias superior. Estos datos corroboran la hipótesis de esos autores y muestran la posibilidad de transferencia de conocimiento de unos dominios a otros pasando por el desarrollo de unas estructuras de razonamiento dotadas de un cierto grado de generalidad.

Además, cabe citar los estudios que sugieren la eficacia de las analogías como estrategia de enseñanza y de aprendizaje. La revisión llevada a cabo por Dagher (1995) parece apoyar la idea de que las analogías constituyen un instrumento de aprendizaje que permite transferir estructuras de conocimiento de unos dominios a otros. Aun con sus limitaciones, su viabilidad pone de manifiesto que, por encima de los conocimientos particulares, los individuos pueden elaborar estructuras de conocimiento de más alto nivel con objeto de comprender el mundo que les rodea. Por cierto, en el contexto educativo de nuestro país aún son muy escasos los trabajos desarrollados en esta línea. Por ello, sería deseable que en el futuro los investigadores de nuestro entorno nos interesásemos más por este tipo de estrategia de enseñanza que parece que puede jugar un papel relevante como instrumento dirigido incluso al cambio conceptual (Brown, 1994; Treagust et al., 1996).

Así mismo, cabe también mencionar nuestra modesta contribución al respecto (Oliva, 1994) en un estudio llevado a cabo sobre la influencia del nivel de desarrollo intelectual en la naturaleza y evolución de las concepciones en mecánica. En él se puso de manifiesto que, a pesar de que, en su globalidad, alumnos adolescentes de alto y bajo nivel de pensamiento formal no difieren de forma drástica en la naturaleza de las ideas que mantienen en una fase previa a la instrucción, la consistencia en el uso de las ideas a través de distintos contextos aumenta a medida que lo hace la capacidad para razonar formalmente. Resultan precisamente los alumnos que manifiestan destrezas generales de razonamiento más avanzadas aquéllos también que muestran un comportamiento más consistente en el uso de sus ideas, erróneas o no, y aquéllos asimismo que logran cambiarlas de un modo más eficiente por el punto de vista científico. En consecuencia, parece razonable pensar que el aprendizaje no sólo se trata de un proceso de transformación en las ideas existentes sino también en una transferencia de aprendizajes de unas situaciones a otras a través de procesos en los que se vean implicadas algunas destrezas o esquemas de razonamiento con un cierto nivel de generalidad.

Por último, cabría comentar el estudio de Gómez-Crespo y otros (1995), que es quizás el que aporta datos más directos al respecto dentro de los que se encuentran cercanos al contexto en el que se inserta este trabajo. En él se pudo comprobar que el grado de comprensión de la idea de conservación de la materia en transformaciones físicas y químicas mejoraba significativamente con el nivel de instrucción y pericia de los sujetos, si bien las diferencias observadas entre estudiantes de la misma edad de químicas y de psicología eran menores de lo esperado. Los autores reseñados interpretan esta circunstancia sugiriendo la posibilidad de que, en estos últimos, ciertos esquemas adquiridos en el dominio de su especialidad pudieran servirles para afrontar tareas situadas en campos alejados de su formación. Ello, de un modo indirecto, habría servido también para hacer que progresasen en aquellas estructuras que son necesarias en el aprendizaje de la química.

En consecuencia, parece lógico dirigir también nuestra atención al cambio en esas estructuras mentales más o menos generales a la hora de abordar la empresa del cambio conceptual. Sólo en la medida en que cambien las pautas de pensamiento que aparecen repitiéndose en distintos ámbitos y se modifiquen también las estructuras que le sirven de soporte, podremos hablar de las condiciones necesarias para que el cambio conceptual en 
dominios específicos tenga lugar. En concreto, en sus trabajos Pozo y otros (1995) asumen la necesidad de superar algunas restricciones cognitivas que se encuentran detrás de esas teorías o modelos implícitos y adquirir las siguientes estructuras que son esenciales para la comprensión de gran parte de los contenidos de la ciencia:

a)interacción de sistemas;

b) cuantificación de relaciones (esquemas de proporcionalidad, probabilidad y correlación);

c) la conservación en sistemas en equilibrio.

Pero a pesar de que la adquisición de estas estructuras sería esencial para el cambio en el pensamiento del alumno, no por ello el éxito quedaría asegurado dada la importancia que tienen también los aspectos conceptuales concretos de cada dominio en particular (Vosniadou, 1994). Así, pues, el cambio en el conocimiento parece comportar un cambio a dos niveles: uno de ellos que implica un cambio a gran escala en las estructuras mentales del individuo, y otro que opera de un modo más fino a través de un cambio en los contenidos específicos a través de los cuales se concretan esas estructuras. En el apartado que sigue hablaremos del cambio conceptual referido a este último nivel, ya que parece que es el que ha estado en la punta de mira de los modelos habituales de cambio conceptual.

\section{Cuestionamiento del cambio como mero desplazamiento de ideas}

Una de las primeras críticas que podríamos hacer al modelo conceptual clásico de Posner y otros es su limitada capacidad para explicar cómo se produce el proceso de cambio en las concepciones. Se detiene más en las condiciones que deben verificarse para que el cambio se produzca que en los mecanismos que tienen lugar mientras el proceso se produce. No deja claro cómo se adquieren las nuevas ideas o cómo se transforman o modifican las ya existentes para dar paso a las nuevas. Tampoco diferencia suficientemente entre sustitución y reestructuración como mecanismos alternativos posibles para explicar el cambio ni se decanta por ninguno de ellos en concreto a la hora de fundamentar sus posturas:

«[...] los marcos de referencia que reconocen los cambios que se producen en el conocimiento no son suficientes para trazar un curso educativo. La investigación también debe intentar comprender cómo se producen esos cambios en la reestructuración del conocimiento y cómo construir un entorno de aprendizaje que facilite esta reestructuración.» (Duschl y Gitomer, 1995, p. 125).

Ello se debe, pensamos, a la dificultad de estos modelos para abordar el problema del análisis y la representación del conocimiento, aspecto que necesariamente debemos atribuir a la propia naturaleza holística del paradigma organicista sobre el cual se sustentan sus posiciones. Se podría decir, utilizando un símil, que la comprensión de la naturaleza y funcionamiento del conocimiento en los modelos de cambio conceptual ha topado con un problema parecido al que sufrió la química anterior al siglo XIX cuando todavía no existía un modelo como el de Dalton que pudiera ayudar a clarificar conceptos tales como el de elemento, compuesto, mezcla, reacción química, etc. Quizás sea ésta una de las razones fundamentales que han hecho que la mirada se haya vuelto hacia modelos de conocimiento y aprendizaje provenientes de áreas próximas a las ciencias cognitivas y a la inteligencia artificial, donde estar o no en posesión de modelos adecuados para la representación explícita del conocimiento ha constituido un problema de supervivencia.

Los problemas de teorización en este terreno arrancan ya de las carencias teóricas observadas habitualmente en los estudios sobre concepciones. En su mayoría, los trabajos en esta línea han carecido de una base psicológica de partida sólida y explícita, moviéndose en un plano puramente descriptivo y apoyándose sobre las propias concepciones personales de los investigadores acerca de las concepciones de los alumnos. No es casual, por tanto, la gran variedad de términos empleados en la literatura didáctica para referirse al conocimiento intuitivo del alumno sobre dominios científicos (Abimbola, 1988; Jiménez et al., 1994), y la gran confusión y diversidad de descriptores que aparecen también para referirse a las distintas propiedades que presentan las concepciones: coherencia, consistencia, estabilidad... (Oliva, 1988b). En el mejor de los casos, la fuentes de fundamentación han provenido del campo de la epistemología de las ciencias, lo cual ha servido como una forma interesante de enriquecimiento, pero también en cierta forma ha supuesto una renuncia a abordar el problema del aprendizaje desde una óptica más específica como la que plantean las situaciones de enseñanza en el aula.

En consecuencia, no debe de extrañar que, en ausencia de un marco teórico suficientemente potente y clarificador, la mayoría de los investigadores hayan llegado a identificar cambio conceptual con desplazamiento de una idea por otra, asociación cuya validez resulta cuanto menos dudosa. La misma idea de sustitución de una concepción intuitiva por otra científica, señalan Larochelle y Désautels (1991), resulta insostenible desde una óptica constructivista, dado que unas y otras no son comparables al no contestar a las mismas preguntas ni responder a los mismos intereses. En este sentido, hay que mencionar positivamente la aportación que Giordan hace al respecto (Giordan, 1989; 1996) en su modelo de cambio conceptual. Este autor sitúa una parte importante de su atención no sólo en las transformaciones globales que se producen en el conocimiento del alumno cuando aprende, sino también en los cambios y conservaciones parciales que acontecen durante el proceso de evolución conceptual. Por ello, contempla el aprendizaje como un proceso complejo en el que se complementan las opciones de conocer «gracias a» (Gagné), «a partir de» (Ausubel), «con» (Piaget) y «en contra de» (Bachelard). En sus conclusiones no difiere sustancialmente del modelo propuesto por Posner y otros, y de hecho las condi- 
ciones para el cambio que desde él se propugnan son similares a las de aquél. Pero se distancia de él en sus fundamentos y en algunos puntos importantes del método de su discurso. Así, el modelo de Giordan hace referencia no sólo a las condiciones del cambio sino que también aporta elementos de reflexión y análisis necesarios para comprender cuáles son los mecanismos a través de los cuales se produce ese cambio. Incluso, llega a aportar una serie de parámetros que potencialmente serían útiles a la hora de acometer la empresa de la representación del conocimiento en la modelización de las concepciones: marco de referencia, red semántica, significantes e invariantes operatorias.

En segundo lugar, debemos reconocer que la idea de sustitución conceptual mantiene ciertas reminiscencias empiristas (Rowell y Dawson, 1988; Abimbola, 1988; Larochelle y Désautels, 1991), en el sentido de que buscan la eliminación de las ideas previas de los alumnos para luego introducir otras nuevas partiendo de cero. Utilizando una metáfora podría decirse que más que un cambio como restauración de un viejo edificio en ruina, parece que lo que propone es el derribo del viejo edificio y el alzamiento de otro nuevo sobre un solar vacío.

Finalmente hay que señalar que las propias condiciones de insatisfacción, inteligibilidad y plausibilidad propuestas en el modelo de cambio conceptual responden a patrones de tipo subjetivo y dependen también de factores afectivos y motivacionales (Pintrich et al., 1993; Villani y Cabral, 1997). Por ello, difícilmente el cambio en las ideas puede llegar a comprenderse siguiendo un esquema tan simplista como lo es el de eliminar una idea y colocar otra distinta en su lugar.

Teniendo en cuenta las causas apuntadas, no debe extrañar que las diferentes experiencias didácticas realizadas sobre cambio conceptual basándose en la idea de desplazamiento o sustitución (Hierrezuelo y Molina, 1988; Bullejos y Sampedro, 1990; Oliva, 1994) hayan detectado niveles de cambio superiores a los observados en la enseñanza por transmisión pero a todas luces todavía insuficientes como suelen reconocer sus propios autores.

En respuesta a la idea de cambio por desplazamiento o sustitución de ideas, autoras como Jiménez-Aleixandre han llamado la atención sobre lo importante que es que los alumnos logren relacionar y diferenciar la naturaleza e implicaciones de diferentes concepciones rivales. Y para ello sugiere la posibilidad de ofrecerles un marco histórico de referencia desde el que establecer tales diferenciaciones, o simplemente hacerles comparar las respuestas que daban al comienzo con las que resultan apropiadas desde el punto de vista científico (JiménezAleixandre, 1991, 1992).

Por otro lado, en oposición a la idea de cambio como sustitución, autores como Linder (1993) o el propio Hewson (Hewson y Hewson, 1992) sostienen que el proceso de cambio conceptual no ha de consistir en un mero desplazamiento de una idea por otra, sino en la distinción entre los contextos en los cuales una idea puede o no resultar útil, de un modo similar a lo que ocurre con los modelos científicos. Linder (1993) nos recuerda que, en la evolución histórica de la ciencia, la aparición de nuevas teorías más completas, como la teoría de la relatividad de Einstein o la mecánica cuántica, no implica el descarte total y el desmantelamiento absoluto de teorías anteriores, como la mecánica newtoniana. No se trataría, pues, de verificar un proceso de sustitución traumática de unas ideas por otras, como de propiciar que ambos sistemas explicativos convivan y que sea el alumno el que controle y decida convenientemente qué argumentos puede y debe usar en cada momento. En consecuencia, el fin de la escuela debería ser el de proporcionar a los estudiantes las capacidades necesarias para distinguir entre diferentes conceptualizaciones de una manera adecuada según el contexto específico (Linder, 1993; Ebenezer y Gaskell, 1995).

Cabe preguntarse ahora cuál será el entorno didáctico más adecuado y las estrategias más acordes con estas nuevas visiones de las ideas de los alumnos y del cambio conceptual. Es a este aspecto al que dedicaremos espacio a continuación mostrando algunas de las críticas de las que han sido objeto las estrategias basadas en el conflicto conceptual, que parecen haber sido las que más incidencia han tenido en las propuestas innovadoras recientes encaminadas a propiciar un cambio conceptual.

\section{Críticas al conflicto conceptual como estrategia sistemática para el cambio en las ideas}

Nos referiremos en concreto al conflicto entendido como contradicción entre las expectativas del alumno basadas en sus concepciones personales y las evidencias empíricas. Aunque compartimos un cierto interés por las estrategias basadas en este tipo de situaciones, y pensamos que pueden ser de cierta utilidad si se utilizan con mesura y en conjunción con otras estrategias de enseñanza, consideramos que no pueden ni deben constituir por sí solas la base de un modelo de enseñanza preocupado por la evolución de las ideas de los alumnos. Para justificar esta opción, a continuación retomamos algunas de las críticas razonadas más importantes de las que han sido objeto los modelos basados en el conflicto conceptual como eje de propuestas didácticas.

La primera de las críticas se apoya en algunos trabajos que sugieren que la aplicación sistemática de estrategias de esta naturaleza no sólo no suelen llegar a modificar las ideas de los alumnos con todo el éxito esperado (Dreyfus et al., 1990), sino que suelen consumir también más tiempo del deseado (Gunstone et al., 1988) y en ocasiones despiertan cierta inhibición y desánimo en los alumnos cuando su uso es reiterado e implican el rechazo sistemático de las ideas previamente expresadas (Furió et al., 1994). Las ideas previas se muestran mucho más resistentes al cambio de lo que en principio era de esperar y, a lo sumo, lo que se consigue es que, tras largos períodos de instrucción, las nuevas ideas convivan y coexistan con las ideas anteriores que no llegan a ser del todo erradicadas. De ahí que autores como 
Gutwill y otros (1996) concluyan que el contraste de pareceres a través de la experiencia no sea suficiente para mejorar el aprendizaje y que sea necesario adiestrar a los alumnos para que sean capaces de aportar explicaciones desde variados puntos de vistas que resulten coherentes entre sí.

La segunda de las críticas se dirige a las serias dudas que se han suscitado en torno al carácter constructivista o no de esta estrategia didáctica. Quisiéramos comentar al respecto que estas estrategias corren el peligro de hacernos olvidar una de las premisas básicas del constructivismo como lo es la de enseñar a partir de lo que el alumno sabe, desplazándose la atención hacia enseñar a partir de lo que el alumno no sabe o se considera que «sabe mal». En este sentido, muchos autores creen hoy factible la idea de cambio a partir de aquellas ideas que el alumno mantiene y que son correctas o parcialmente correctas dentro del contexto escolar, y que pueden utilizarse como punto de anclaje para el aprendizaje o la ampliación de ideas (Clement et al., 1989). El propio Hewson (1981) recogía ya como vimos esta modalidad de cambio dentro de su noción de captura conceptual, y las analogías vuelven hoy a cobrar actualidad en la investigación didáctica, como lo demuestra la sugerente revisión de Dagher (1995).

En respuesta a las críticas comentadas parecen haber surgido estrategias alternativas al conflicto cognitivo como motor del cambio conceptual. En algunos casos, estas otras orientaciones han tratado de aproximar la enseñanza y aprendizaje de las ciencias en el aula al proceso de construcción de conocimientos en la comunidad científica, en lugar de hacerlo tomando como eje procesos de desequilibración cognitiva de las ideas preexistentes. Entre ellas cabe citar la perspectiva propuesta por Duschl (1995) en sus trabajos sobre el proyecto SEPIA o también el modelo de enseñanza por investigación (Gil, 1996; Furió et al., 1994). Estos modelos comparten un interés por implicar a los alumnos no sólo en la tarea de utilizar el conocimiento científico, sino también en las estrategias propias de la ciencia a la hora de abordar problemas.

En concreto, en el caso del modelo de Gil, el cambio conceptual ni siquiera se convierte en un objetivo en sí mismo, sino más bien en una consecuencia de un cambio originado en otros niveles que involucran al plano procedimental y actitudinal del alumno. El motor de inferencia de ese modelo descansa sobre la analogía del alumno como investigador novel. Estos autores critican las estrategias de cambio conceptual como confrontación de ideas por considerarlas carentes de un contexto didáctico en el que cobre sentido todo el proceso de explicitación y cuestionamiento de las concepciones espontáneas y en la introducción de otras nuevas. En su modelo, estos autores proponen no tanto el cambio conceptual en sí mismo, mediante el rechazo de las concepciones de partida, como el planteamiento y resolución de situaciones problemáticas abiertas que generen cierto interés para el estudiante y que sean resueltas en un entorno colaborativo y dirigido mediante aplicación de estrategias habituales en el marco de trabajo de los científicos: delimitación del problema, formulación de hipótesis, etc. La clarificación e implementación del modelo a través de propuestas didácticas concretas viene constituyendo en nuestro país una muestra de todo un programa de investigación, y los resultados obtenidos parecen ser esperanzadores, como lo muestran algunos estudios sobre cambio conceptual realizados dentro de este marco educativo (Carrascosa, 1987; Bullejos y Sampedro, 1990; Oliva, 1994; Varela y Martínez-Aznar, 1997). No obstante, conviene señalar que, si antes decíamos que los mecanismos del cambio conceptual están aún por aclarar, con más razón ahora hemos de reconocer que las carencias teóricas respecto al cambio procedimental y actitudinal son todavía más notorias. Ello no debiera de extrañar dado que, hasta ahora, los investigadores sobre didáctica de las ciencias hemos estado preocupados por problemas relacionados con el aprendizaje de conceptos científicos, prestando menos atención a problemas relativos a la adquisición de procedimientos y actitudes. Resultado de ello es que aún no se pueda hablar de modelos que expliquen el cambio procedimental o el cambio actitudinal en ciencias, ni siquiera a un nivel semejante al que Posner y otros (1982) adoptan en su modelo sobre el cambio en los conceptos científicos.

Por otra parte, en un estudio reciente, Pozo y otros (1995) retoman algunas contribuciones surgidas en trabajos de otros autores e identifican otra vía de cambio basada en la instrucción a través de modelos. Difiere de los modelos basados en el conflicto conceptual en que ahora el debilitamiento de las ideas intuitivas, y su diferenciación de las teorías científicas, se produce a través de las contradicciones que el alumno observa en sus razonamientos cuando compara los argumentos que se derivan de sus ideas previas y de los modelos científicos que han aprendido; y, sobre todo, a través de la consistencia y organización interna que detectan en las ideas científicas en comparación con su punto de vista intuitivo. El metaconocimiento reemplaza al descubrimiento dirigido como herramienta de búsqueda de la validez de los argumentos, y los procesos de asimilación del nuevo conocimiento se implementan mediante estrategias de instrucción directa, lo que no debe entenderse según Pozo y otros (1995) como un regreso a los métodos de enseñanza tradicionales. En palabras del propio Pozo «[...] la instrucción debe partir de un modelo de cambio conceptual, y tras la presentación de un modelo o teoría, seguir los pasos o fases necesarios para alcanzar el cambio conceptual» (Pozo et al., 1995, p. 173). Con ello la noción de conflicto adquiere una dimensión diferente, y ahora sirve como referente de organización interna y no tanto como fuente de desestabilización a partir de referentes externos al individuo, aunque éstos evidentemente también sean necesarios.

Si bien la validez de este otro enfoque está por demostrar y aún no tenemos datos de su eficacia en propuestas instruccionales concretas, pensamos que deja aún algunos aspectos por aclarar. Deja en el aire, por ejemplo, detalles importantes como cuál ha de ser el enfoque más adecuado a seguir durante el proceso de instrucción y cómo ello afectaría al papel del profesor y del alumno en el aula o a la propuesta de tareas de aprendizaje. Además, 
desde un punto de vista constructivista no llega a resolver los problemas epistemológicos que también planteaban las estrategias basadas en el conflicto conceptual a través de la experiencia sensible, ni deja claro cuáles son los mecanismos de asimilación del nuevo conocimiento. Dicho de otro modo, no nos aclara cómo los alumnos conseguirán dar un significado adecuado al modelo científico que se les presenta, teniendo en cuenta que, como diría Giordan (1996), han de hacerlo «gracias a», «a partir de», «con» y «en contra de» de un conjunto de concepciones alejadas del punto de vista científico. Para finalizar diremos que tampoco ofrece detalles acerca de cómo articular en la enseñanza el dominio conceptual, el metodológico y el actitudinal, todo lo cual no hace sino añadir nuevas incertidumbres a la problemática en cuestión. Sería deseable que en el futuro próximo los partidarios de estas propuestas clarificasen sus posturas y para ello la mirada la dirigiesen a los procesos de asimilación y no sólo a los de acomodación y reestructuración, como mecanismos claves que permiten explicar de qué manera se verifica el cambio; o mejor aún, cómo ciertas ideas afines a la ciencia escolar van progresivamente siendo incorporadas y preferidas por los alumnos en comparación con sus ideas intuitivas iniciales.

\section{CONCLUSIONES}

En los últimos años han surgido algunas novedades importantes en el estudio de las concepciones. En concreto, hemos descrito y fundamentado cuatro principios básicos que vienen orientando algunos de los estudios realizados últimamente en este terreno y que encuentran fundamento en los trabajos centrados en las ideas de los alumnos como teorías implícitas o como modelos mentales. Estos principios son el de estructuración implícita, el de diversidad-coexistencia, el de sistematicidadhomogeneidad limitada y el de probabilidad.

Así mismo, hemos mencionado qué implicaciones parecen tener estas nuevas visiones en los modelos de cambio conceptual. En especial nos hemos centrado en describir algunas de las críticas de las que han sido objeto los modelos de cambio basados en el desplazamiento de ideas y en el conflicto conceptual como confrontación de ideas. Nuestra opinión es que la mayoría de las críticas vertidas tienen en su origen la escasa capacidad de estos modelos para esclarecer cuáles son los procesos internos que se producen durante el cambio de una idea por otra, dado que se han centrado en las condiciones del cambio pero han aclarado poco acerca de los mecanismos que a través del mismo operan. Como ya advertimos en su momento, los modelos de cambio conceptual adolecen de la falta de una visión analítica que les permita comprender cuál es el entramado de ideas que compone el tejido del conocimiento; o dicho de otro modo, carecen de procedimientos que posibiliten acceder a un nivel de representación que ayude a comprender cómo se estructura y organiza el conocimiento y qué cambios sufre durante los procesos de aprendizaje. De ahí que algunos autores y autoras hayan buscado estrategias de representación de las concepciones para modelizar los procesos de cambio conceptual, basándose en mapas conceptuales (Dykstra et al., 1992) o en modelos mentales (Gutiérrez y Ogborn, 1992), y que incluso exista un creciente interés por parte de los psicólogos cognitivos por abordar problemas de corte epistemológico. De todo ello se desprende la necesidad de revisar nuestras ideas acerca del cambio conceptual como modelo de aprendizaje y de las iniciativas didácticas que a partir del mismo se pueden inferir. Así mismo, parece conveniente orientar la investigación en este campo tomando en cuenta las nuevas aportaciones surgidas, en especial de aquéllas que puedan ayudarnos a analizar el conocimiento de los alumnos, descubrir regularidades y comprender cómo se articulan las distintas piezas que integran la estructura cognitiva. Sólo en la medida en que seamos capaces de conectar el nivel de análisis y de síntesis de nuestro estudio, podremos comprender cómo funcionan las ideas, cómo cambian con la instrucción y cómo seríamos capaces de hacer que evolucionen a través de la enseñanza.

En adelante, creemos necesario que las propuestas didácticas constructivistas asuman e incorporen de alguna forma los presupuestos teóricos que hemos revisado y fundamentado a lo largo del trabajo. No obstante, las implicaciones didácticas de estos presupuestos son hoy por hoy difíciles de precisar, dado que los mecanismos de cambio permanecen aún inciertos. Es posible que en un futuro más o menos próximo la didáctica de las ciencias pueda disponer de modelos de aprendizaje de conceptos más apropiados y precisos que los formulados hasta ahora. Y es de esperar que ello arroje luz sobre las estrategias de enseñanza más convenientes para hacer que las ideas de los alumnos evolucionen de un modo eficiente.

De momento, sea cual sea la opción didáctica por la que nos decantemos a la hora de abordar la enseñanza -conflicto conceptual, instrucción directa, analogías, resolución de problemas o una combinación de todas ellas-, deberíamos actuar con suficiente cautela y siempre contando con la actividad metacognitiva del alumno como elemento esencial y regulador del proceso. Sólo en la medida que el alumno se encuentre comprometido, cognitiva y afectivamente, con lograr hacer evolucionar sus concepciones, podremos esperar un cierto éxito en nuestra tarea de enseñar ciencias (Pintrich et al., 1993).

\section{AGRADECIMIENTOS}

La investigación en la que se basa el contenido de este trabajo ha sido aprobada y financiada por el Ministerio de Educación y Cultura a través de la convocatoria del CIDE de ayudas a la investigación educativa. Expresamos desde aquí nuestro agradecimiento a dicho organismo. 


\section{REFERENCIAS BIBLIOGRÁFICAS}

ABIMBOLA, I.O. (1988). The problem of terminology, in the study of student conception in science. Science Education, 72(2), pp. 175-184.

ACEVEDO, J.A., BOLÍVAR, J.P., LÓPEZ-MOLINA, E.J. y TRUJILLO, M. (1989). Sobre las concepciones en dinámica elemental de los adolescentes formales y concretos y el cambio metodológico. Enseñanza de las Ciencias, 7(1), pp. 27-34.

ADEY, P. (1992). The CASE results: implications for science teaching. International Journal of Science Education, 14(2), pp. 139-146.

ADEY, P. y SHAYER, M. (1990). Accelerating the development of formal thinking in middle and high school students. Journal of Research in Science Teaching, 27(3), pp. 267-285.

ANDERSSON, B. (1986). The experiential gestalt of causation: a common core to pupils' preconceptions in Science. European Journal of Science Education, 8(2), pp. 155-171.

BLANCO, A., y PRIETO, T. (1997). Pupils' views on how string and temperature affect the dissolution of a solid in a liquid: a cross-age study (12 to 18). International Journal of Science Education, 19(3), pp. 303-316.

BROWN, D.E. (1994). Facilitating conceptual change using analogies and explanatory models. International Journal of Science Education, 16(2), pp. 201-214.

BULLEJOS, J., y SAMPEDRO, C. (1990). Diferenciación de los conceptos de masa, volumen y densidad en los alumnos de BUP mediante estrategias de cambio conceptual. Enseñanza de las Ciencias, 8(1), pp. 31-36.

CAREY, S. (1991). Knowledge acquisition: Enrichment or conceptual change?, en Carey, S. y Gelman, R. (eds.), The epigenesis of mind. Hillsdale, Nueva Jersey: Erlbaum.

CARRASCOSA, J.(1987).Tratamiento didáctico en la enseñanza de las ciencias de los errores conceptuales. Tesis doctoral no publicada. Universidad de Valencia.

CLEMENT, J., BROWN, D., y ZIETSMAN, A. (1989). Not all preconceptions are misconceptions: finding anchoring conceptions' for grounding instruction on students' intuitions. International Journal of Science Education, 11(5), pp. 554-565.

CHI, M. (1992) Conceptual change within and across ontological categories: Examples from learning and discovery in science, en Giere, R. (ed.), Cognitive models of Science: Minnesota studies in the philosophy of science. Minneapolis, MN: University of Minnesota Press.

CLOUGH, E. y DRIVER, R. (1986). A study of consistency in the use of students' conceptual framework across different task contexts. Science Education, 70(4), pp. 473-493.

DAGHER, Z.R. (1994). Does the use of analogies contribute to conceptual change? Science Education, 78(6), pp. 601-614.

DAGHER, Z.R. (1995). Review of studies on the effectiveness of instructional analogies in science education. Science Education, 79(3), pp. 295-312.

DREYFUS, A., JUNGWIRTH, E. y ELIOVITCH, R. (1990). Applying the «cognitive conflict» strategy for conceptual change-some implications, difficulties and problems. Science Education, 74(5), pp. 555-569.
DUSCHL, R.A., y GITOMER, G. (1995). Perspectivas epistemológicas sobre el cambio conceptual: implicaciones para la práctica educativa. Comunicación, lenguaje y educación, 25, pp. 107-125.

DUSCHL, R. A. (1995). Más allá del conocimiento: los desafíos epistemológicos y sociales de la enseñanza mediante el cambio conceptual. Enseñanza de las Ciencias, 13(1), pp. 3-14.

DYKSTRA, D.I., BOYLE, C.F., y MONARCH, I.A. (1992). Studying conceptual change in learning physics. Science Education, 76(6), pp. 615-652.

EBENEZER,J.V. y GASKELL,P.J.(1995). Relational conceptual change in solution chemistry. Science Education, 79(1), pp. $1-17$.

FURIÓ, C., BARRENETXEA, I. y REYES, J.V. (1994). Contribución de la resolución de problemas como investigación al paradigma constructivista de aprendizaje de las ciencias. Investigación en la Escuela, 24, pp. 88-99.

GALILI, I. y BAR, V. (1997). Children's operational knowledge about weight. International Journal of Science Education, 19(3), pp. 317-340.

GIL, D. (1996). New trends in science education. International Journal of Science Education, 18(8), pp. 809-901.

GIORDAN, A. (1989). De las concepciones de los alumnos a un modelo de aprendizaje alostérico. Investigación en la Escuela, 8, pp. 3-14.

GIORDAN, A. (1996). ¿Cómo ir más allá de los modelos constructivistas? La utilización didáctica de las concepciones de los estudiantes. Investigación en la Escuela, 28, pp. 7-22.

GÓMEZ-CRESPO, M.A., POZO, J.I. y SANZ, A. (1995). Students' ideas on conservation of matter: effects of expertise and context variables. Science Education, 79(1), pp. 77-93.

GRECA, I.M. y MOREIRA, M.A. (1997). The kinds of mental representations - models, propositions and images- used by college physics students regarding the concept of field. International Journal of Science Education, 19(6), pp. 711-724.

GUNSTONE, R., WHITE, R. y FENSHAM, P. (1988). Developments in style and purpose of research on the learning of science. Journal of Research in Science Teaching, 25, pp. 513-530.

GUTIÉRREZ, R. (1990). Aportaciones de la investigación en inteligencia artificial a la investigación didáctica: el modelo mental mecánico de Kleer y Brown, en Aspectos didácticos de la física y química. ICE de la Universidad de Zaragoza.

GUTIÉRREZ, R. y OGBORN, J. (1992). A causal framework for analysing alternative conceptions. International Journal of Science Education, 14(2), pp. 201-220.

GUTWILL, J., FREDERIKSEN, J. y RANNEY, M. (1996). Seeking the causal connection in electricity: shifting among mechanistic perspectives. International Journal of Science Education, 18(2), pp. 143-162.

HARRISON, A.G. y TREAGUST, D.F. (1996). Secondary students' mental models of atoms and molecules: implications for teaching chemistry. Science Education, 80(5), pp. 509-534.

HEWSON, P.W. (1981). A conceptual change approach to learning science. European Journal of Science Education, 34, pp. 383-396. 
HEWSON, P.W. y BEETH, E. (1995). Enseñanza para un cambio conceptual: ejemplos de fuerza y de movimiento. Enseñanza de las Ciencias, 13(1), pp. 25-35.

HEWSON, P. y HEWSON, M. (1992). The status of student conceptions, en Duit, R., Golberg, F. y Niedderer, H. (eds.), Research in physics learning: Theoretical issues andempirical studies. Kiel, Alemania: Institute of Science Education.

HEWSON, P.W. y THORLEY, N.R. (1989). The conditions of conceptual change in the classroom. International Journal of Science Education, 11, pp. 541-553.

HIERREZUELO, J. y MOLINA, E. (1988). La influencia de las ideas previas en el proceso de enseñanza-aprendizaje. Un ejemplo: la formación del concepto de fuerza en $2^{\circ}$ de BUP. Investigación en la Escuela, 4, pp. 49-57.

JIMÉNEZ, E., SOLANO, I. y MARÍN, N. (1994). Problemas de terminología en estudios realizados acerca de «lo que el alumno ya sabe» sobre ciencias. Enseñanza de las Ciencias, 12(2), pp. 235-245.

JIMÉNEZ-ALEIXANDRE, M.P. y FERNÁNDEZ, J. (1989). ¿Han sido seleccionados o se han acostumbrado? Ideas de los estudiantes de biología sobre selección natural y consistencia entre ellas. Infancia y Aprendizaje, 47, pp. 67-81.

JIMÉNEZ-ALEIXANDRE, M.P. (1991). Cambiando las ideas sobre el cambio biológico. Enseñanza de las Ciencias, 9(3), pp. 248-256.

JIMÉNEZ-ALEIXANDRE, M.P. (1992). Thinking about theories or thinking with theories?: a classroom study with natural selection. International Journal of Science Education, 14(1), pp. 51-61.

KEYS, C.W. (1997). An investigation of the relationship between scientific reasoning, conceptual knowledge and model formulation in a naturalistic setting. International Journal of Science Education, 19(8), pp. 957-970.

KHUN, D., SCHAUBLE, L., y GARCÍA-MILA, M. (1992). Cross-domain development of scientific reasoning. Cognition and Instruction, 9, pp. 285-327.

LAROCHELLE, M. y DÉSAUTELS, J. (1991). «Of course, it's just obvious»: adolescents' ideas of scientific knowledge. International Journal of Science Education, 13(4), pp. 373-389.

LAWSON, A.E. y THOMPSON, L.D. (1988). Formal reasoning ability and misconceptions concerning genetics and natural selection. Journal of Research in Science Teaching, 25(9), pp. 733-746.

LICHT, P. (1987). A strategy to deal with conceptual and reasoning problems in introductory electricity education. Proceedings of the Second International Seminar: Misconceptions and educational strategies in science and mathematics. Cornell University: Ithaca.

LINDER, C.J. (1993). A challenge to conceptual change. Science Education, 77(3), pp. 293-300.

LINJSE, P. (1990). Energy between the life-world of pupils and the world of physics. Science Education, 74(5), pp. 571-583.

LUFFIEGO, M., BASTIDA, M.F., RAMOS, F. y SOTO, J. (1994). Systemic model of conceptual evolution. International Journal of Science Education, 16(3), pp. 305-313.

MALONEY, D.P. y SIEGLER, R.S. (1993). Conceptual competition in physics learning. International Journal of Science Education, 15(3), pp. 283-295.
MARTON, F. (1981). Phenomenography-describing conceptions of the world around us. Instructional Science, 10, pp. 177-200.

MONK, M. (1995). On the identification of principles in science that might inform research into students' beliefs about natural phenomena. International Journal of Science Education, 17(5), pp. 565-573.

MOREIRA, M.A. (1997). Modelos mentais. Investigaçoes em Ensino de Ciências, 1(3) [on line]. Disponible en: http:// www.if.ufrgs.br/public/ensino/revista.html.

NÚÑEZ, F. y BANET, E. (1996). Modelos conceptuales sobre las relaciones entre digestión, respiración y circulación. Enseñanza de las Ciencias, 14(3), pp. 261-278.

NUSSBAUM, J. (1989). Classroom conceptual change: philosophical perspectives. International Journal of Science Education, 11, pp. 530-540.

OLIVA, J.M. (1994). Influencia de las variables cognitivas en la construcción de conocimientos de mecánica. Un estudio empírico y un análisis computacional. Tesis doctoral no publicada. Universidad Nacional de Educación a Distancia. Madrid.

OLIVA, J.M. (1996). Estudios sobre consistencia en las ideas de los alumnos en ciencias. Enseñanza de las Ciencias, 14(1), pp. 87-92.

OLIVA, J.M. (1998a). Sobre la estabilidad de las concepciones de los alumnos en física, en Jiménez, R. y Wamba, A. (eds.), Avances en didáctica de las ciencias experimentales. Universidad de Huelva.

OLIVA, J.M. (1998b). Estatus de las concepciones de los alumnos en física: un proyecto de investigación en curso, en Investigación e Innovación en la Enseñanza de las Ciencias (En prensa).

PINTÓ, R., ALIBERAS, J. y GÓMEZ, R. (1996). Tres enfoques en la investigación sobre concepciones alternativas. Enseñanza de las Ciencias, 14(2), pp. 221-232.

PINTRICH, P.R., MARX, R.W. y BOYLE, R. (1993). Beyond cold conceptual change: The role of motivational beliefs and classroom contextual factors in the process of conceptual change. Review of Educational Research, 63(2), pp. 167-199.

POSNER, G., STRIKE, K., HEWSON, P. y GERTZOG, W. (1982). Accomodation of a scientific conception: Toward a theory of conceptual change. Science Education, 66(2), pp. 211-227.

POZO, J.I., GÓMEZ CRESPO, M.A. y LIMÓN, M. y SANZ, A. (1991). Procesos cognitivos en la comprensión de la ciencia: las ideas de los adolescentes sobre la química. Madrid: CIDE.

POZO, J.I., PÉREZ, M.P., SANZ, A. y LIMÓN, M. (1992). Las ideas de los alumnos sobre la ciencia como teorías implícitas. Infancia y Aprendizaje, 57, pp. 3-22.

POZO, J.I., SANZ, A. y GÓMEZ CRESPO, M.A. (1995). Cambio conceptual: del conocimiento personal al conocimiento científico, en Aspectos didácticos de física y química (física). Instituto de Ciencias de la Educación. Universidad de Zaragoza.

RODRÍGUEZ, A., RODRIGO, M.J. y MARRERO, J. (1993). El proceso de construcción del conocimiento. Teorías implícitas o teorías científicas, en Rodrigo, M.J. et al. (comp.), Las teorías implícitas. Una aproximación al conocimiento cotidiano. Madrid: Aprendizaje-Visor. 
RODRÍGUEZ, A. y GONZÁLEZ, R. (1995). Cinco hipótesis sobre las teorías implícitas. Revista de Psicología General y Aplicada, 48(3), pp. 221-229.

ROGAN, J.M. (1988). Development of a conceptual framework of heat. Science Education, 72(1), pp. 103-113.

ROWELL, J.A. y DAWSON, C.J. (1989). Towards an integrated theory and practice for science teaching. Studies in Science Education, 16, pp. 47-73.

ROZIER, S. y VIENNOT, L. (1991). Students' reasonings in thermodynamics. International Journal of Science Education, 13(2), pp. 159-170.

SHAYER, M. y ADEY, P. (1992). Accelerating the development of formal thinking in middle and high school students III: testing the performance of effects. Journal of Research in Science Teaching, 29(10), pp. 1101-1115.

SCHAUBLE, L., KLOPFER, L, y RAGHAVEN, K. (1991). Students' transition from an engineering model to a science model of experimentation. Journal of Research in Science Teaching, 28, pp. 859-882.

STAVY, R. y TIROSH, D. (1996). Intuitive rules in science and mathematics: the case of «more of A-more of B». International Journal of Science Education, 18(6), pp. 653-667.

TIROSH, D. y STAVY, R. (1996). Intuitive rules in science and mathematics: the case of «everything can be divided by two». International Journal of Science Education, 18(6), pp. 669-683.
TREAGUST, D.F., HARRISON, A.G., y VENVILLE, G.J. (1996). Using an analogical teaching approach to engender conceptual change. International Journal of Science Education, $18(2)$, pp. $213-229$

TRUMPER, R. y GORSKY, P. (1993). Learning about energy: the influence of alternative frameworks, cognitive levels, and closed-mindedness. Journal of Research in Science Teaching, 30(7), pp. 637-648.

VARELA, P. y MARTÍNEZ-AZNAR, M.M. (1997). Una estrategia de cambio conceptual en la enseñanza de la física: la resolución de problemas como actividad de investigación. Enseñanza de las Ciencias, 15(2), pp. 173-188.

VIENNOT, L. y KAMINSKI, W. (1991). Participation des maîtres aux modes de raisonnement des élèves. Enseñanza de las Ciencias, 9(1), pp. 3-9.

VILLANI, A. y CABRAL, T.C. (1997). Mudança conceitual, subjetividade e psicanálise. Investigaçoes em Ensino de Ciências, 2(1) [on line]. Disponible en: http://www.if.ufrgs.br/ public/ensino/revista.html.

VOSNIADOU, S. (1994). Capturing and modelling the process of conceptual change. Learning and Instruction, 4(1), pp. 45-69.

WATTS, M. y TABER, K.S. (1996). An explanatory gestalt of essence: students' conceptions of the «natural» in physical phenomena. International Journal of Science Education, 18(8), pp. 939-954.

[Artículo recibido en diciembre de 1997 y aceptado en junio de 1998.] 